\title{
FACIAL AND SHOULDER ELECTROMYPGRAPHIC RESPONSE TO HEAT STIMULATION AT DISTINCT INTENSITY LEVELS
}

\author{
F. M. Lima*, S. Walter**, H. C. Traue**, and A. O. Andrade* \\ * Centre for Innovation and Technology Assessment in Health (NIATS), Postgraduate Program in \\ Electrical and Biomedical Engineering, Faculty of Electrical Engineering, Federal University of \\ Uberlândia, Uberlândia, Brazil \\ ** Emotion Lab, Department of Psychosomatic Medicine and Psychotherapy, Ulm University, Ulm, \\ Germany \\ e-mail: fermaxlima@hotmail.com
}

\begin{abstract}
Reliable objective pain assessment is necessary to provide adequate pain relief treatments, especially in case of non-communicative patients. In this sense we address the use of electromyography (EMG) in the context of experimental pain induction. Thus, we aimed to analyze whether thermal painful stimulation can elicit stable electromyographic responses among several random stimulations for the Corrugator, Zygomaticus and Upper Trapezius muscles. Bipolar differential surface EMG were acquired with a sampling rate of $512 \mathrm{~Hz}$, gain of 24, and ADC resolution of 24 bits. Raw data were processed in Matlab with $4^{\text {th }}$ order Butterworth bandpass $(5-256 \mathrm{~Hz})$ filter and $20^{\text {th }}$ order polynomial filters. Then, the Hilbert transform was used to estimate the instantaneous amplitude, which was processed by a thresholding algorithm, so that only responses with prominences larger than $5 \%$ of the entire signal's range were counted. Among Corrugator, Zygomaticus and Trapezius muscles, Zygomaticus was the muscle in which the responses were more often identified (over 47\%), regardless of the stimulation intensity. Thermal painful stimulation at the painful threshold level elicited electromyographic responses in only $20.7 \%$ (Corrugator), 31.3\% (Zygomaticus) and $32.7 \%$ (Trapezius) of the total number of stimuli, while thermal painful stimulation at the tolerance level elicited electromyographic responses in $56.2 \%, 69.8 \%$, and $54.2 \%$ of the total number of stimuli, respectively. We observed that the higher the stimulation intensity, the larger the recognition rates of electromyographic responses for all the three muscles. We concluded that, although using randomized time and intensity stimulation design, we didn't get stable EMG responses with respect to thermal pain stimulation.

Keywords: Biomedical Signal Processing, Electromyography, Pain Measurement.
\end{abstract}

\section{Introduction}

Reliable objective pain assessment is relevant to provide adequate relief for those who suffer, which is not often a problem when patients can properly describe their own pain sensation. In case of non-communicative patients such as young children and adults with communication impairments or in unconscious states, physiological and behavioral scales are usually implemented, which relies on the analysis and description of patient's status from the perspective of a third person. These scales can be imprecise, biased and time consuming, leading to under-treatment of patients' condition and unnecessary suffering, overdose and lifethreatening conditions due to cardiac and respiratory depression. Personal distress of allied health professionals due to the repeated assessment of several inpatients in a busy service is also an issue to take in account.

To address the problem of an objective assessment and continuous monitoring of pain sensation, several methods have been proposed and tested in the literature, ranging from single biosignal measurements to brain imagery and multimodal data fusion [1], each of them with its pros and cons when compared to other commonly used scales.

In this work we address the problem of using electromyographic (EMG) data from facial muscles related to painful facial expressions and cervical muscles related to stressful conditions in an experimental design analysis.

As EMG activity can be an indicator of psychophysiological arousal and pain-related facial expressions are expected to elicit Corrugator and Zygomaticus activation, an increase in Corrugator, Zygomaticus and Trapezius EMG activities is expected. Thus, we aimed to analyze whether thermal painful stimulation can elicit stable electromyographic responses among several random painful stimulations for these three muscles.

\section{Materials e methods}

This study was developed in cooperation between the Centre for Innovation and Technology Assessment in Health (NIATS, Postgraduate Program in Electrical and Biomedical Engineering, Faculty of Electrical Engineering, Federal University of Uberlândia, Uberlândia, Brazil), and the Emotion Lab (Department of Psychosomatic Medicine and Psychotherapy, University Ulm, Ulm, Germany). Electromiographic 
data from the German-based Biovid Pain Database were used (Ethics Committee Approval: 196/10-UBB/bal) [2].

Pain induction design - Each participant was tested for pain threshold and pain tolerance levels with regards to thermal stimulation over the postero-distal surface of the right forearm using a Medoc Pathway Thermode (PHATWAY, Medoc, Israel) four times prior to the main experiment. For instance, pain levels were determined by the highest attempt. Thereafter, two equally distributed intermediate levels were computed prior to the commencement of the experiment. Then, each of the four levels were randomly applied to the participant's forearm 20 times, and sustained for four seconds, with random intervals of 8 to 12 seconds before a new stimulation. More detailed information on the experiment design can be found in [2].

Data acquisition - Surface EMG data from right Corrugator and Zygomaticus facial muscles and from right Upper Trapezius muscle were acquired using differential bipolar sensor configuration by means of a NeXus-32 Amplifier, with a sampling rate of $512 \mathrm{~Hz}, 24$ bits ADC resolution, gain of 24, typical CMRR over 90 $\mathrm{dB}$, and inter-electrode distance of $20 \mathrm{~mm}$.

Data analysis - Raw data was processed in Matlab R2015a (MathWorks Inc., USA), and they were filtered with $4^{\text {th }}$ order bandpass Butterworth filter, and $20^{\text {th }}$ order polynomial filter. Then, the Hilbert transform was used to estimate the instantaneous amplitude. After visual inspection, smoothed data were thresholded to identify prominences higher than $5 \%$ of the total signal's amplitude range within a window of 2 to 8 seconds after stimulus' onset for Corrugator and Zygomaticus muscles, and a window of 2 to 10 seconds after stimulus' onset for the Trapezius muscle. Window's length was arbitrary chosen after visual inspection of the data. Thus, we looked for a time-window in which most of responses occurred for each muscle within this database.

Statistical analysis - All statistics were also computed using Matlab R2015a software (MathWorks ${ }^{\circledR}$, USA). The amount of identified responses was presented in percentages for each participant, and then, average values were computed per stimulation intensity among all participants. Bootstrap technique with 1000 resampling groups was used to compute $95 \%$ confidence intervals. One-way Anova and multcompare visualization function were used to compare different stimulation intensities and also to compare overall response rates of each muscle.

\section{Results}

Results are described in percentages and 95\% confidence intervals for the means. Stimulus' intensity is named of $\mathrm{P} 1$ to $\mathrm{P} 4$, according to ascending intensities from Threshold level (P1) to Tolerance level (P4).

Figure 1 shows the mean response rates and its $95 \%$ confidence interval for each stimulation intensity category and for each muscle. Average response rates are also presented for each muscle, regardless to the stimulation intensity.

Overall average response rates were significant higher when comparing Zygomaticus and Trapezius against Corrugator, but with no significant difference between Zygomaticus and Trapezius (33.7\%, 47.5\%, and $42.6 \%$, respectively, $\mathrm{p}=0.0014$ ).
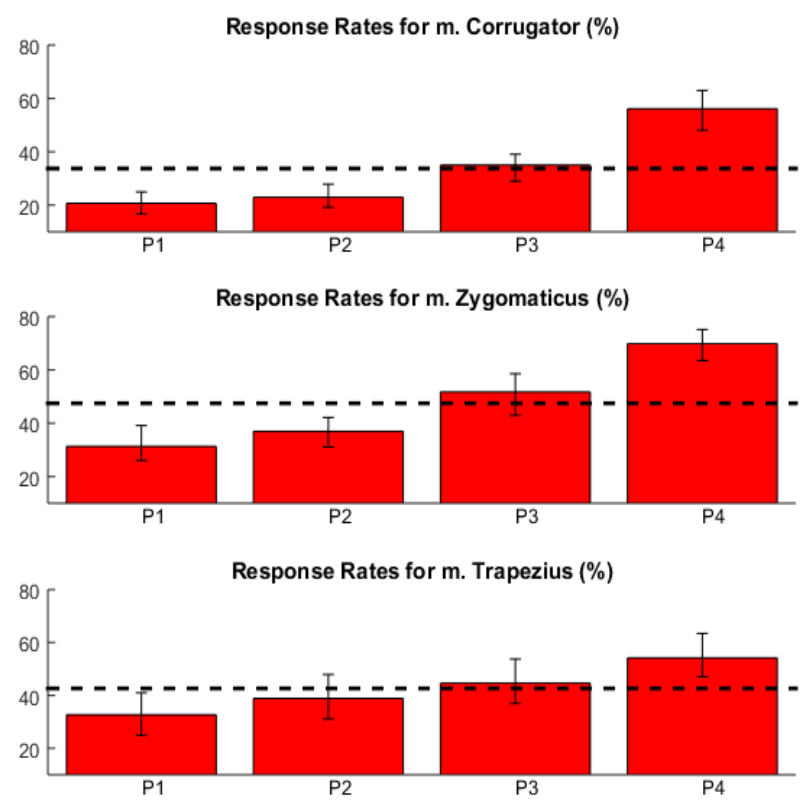

Figure 1: Mean response rate detection and its 95\% confidence interval (using bootstrap technique) by stimulation intensity. Dashed lines are the overall average response rates for each muscle, regardless to the stimulation intensity.

\section{Discussion}

Studying muscle reactivity to external painful stimulation seems to be a reasonable approach to get pain-related information rather than stress-related information due to any other circumstances. This statement is supported by the work of Luijcks and colleagues [11]. They assessed Trapezius EMG activity in the context of an unpredictable and uncontrollable noxious (thus, somehow painful) stimulation. First of all, they showed that expectation of a noxious stimulation increases Trapezius activity when compared to relaxed rest measurements, and additionally, immediate EMG responses were observed just after the stimulation with significant increased activity when compared to pre-stimulation phase. Further, they also pointed out that stressed individuals might show stronger responses after stimulation than less stressed individuals.

On the other side, facial expressions have been used for many years as a source of information on pain sensation intensity, and most of behavioral scales take them into account to allow for the assessment of pain sensation in non-communicative patients [3]. 
Nowadays, advancements in the processing of biomedical signals and image and video data are looking for clues to enable automated pain recognition and grade [1], [4].

Several clinical and experimental studies present positive results in differentiating painful from nonpainful states by means of EMG analysis from facial muscles [5]-[7] and from neck muscles [8], [9]. However, contrary to facial muscle EMG analysis, which enable to get information regarding pain in distant body segments, almost all other EMG research on pain measurement are related to pain in the surrounding areas of one specific muscle.

In our work we did not intend to compare painful versus non-pain status. Instead, we intended to identify how often we get recognizable changes after a painful stimulus in a random stimulation design. Surprisingly, even at higher stimulus' intensity, thermal induced painful stimulation did not elicit significant changes in EMG activity all the time. Highest response rates were found for Zygomaticus, which reached $69.8 \%$, while lowest response rates were $20.7 \%$ for Corrugator.

Contrary to our findings, Wolf and colleagues found more significant responses in Corrugator than in Zygomaticus while using laser-based painful stimulation and a larger set of facial EMG channels [5]. On the other hand, Reicherts and colleagues [10], reported a slight tendency of stronger activation of Zygomaticus during painful stimulation accompanied by no changes in Corrugator activity in the context of painful thermal stimulation.

With regards to experimental pain induction studies, these results might mean at least four different things, which should be further addressed in later studies: (a) the implemented methodology is not sensitive to identify minor electromyographic responses due to painful stimulation; (b) healthy participants may sustain small amounts of pain without significant responses in electromyography; (c) despite using randomized intervals and intensities, an accommodation effect on both signals and pain perception mechanisms may occur along the experiment duration (approximately 30 minutes long), and; (d) as pain is an unpleasant sensation, participants may lie on their calibration levels so that inaccurate measures are taken, and data analysis is strongly affected especially during lower stimulation intensities.

The usage of the amplitude range as a parameter for the estimation of response thresholds may mask participants with low electromyographic activation leading to an under- or overestimation of signals' response rates. Threshold techniques derived from the baseline signal may lead to different results. Furthermore, slow increases in muscle tension as those related to stress and to increase in sympathetic tone are not taken into account. Additionally, a series of stimuli over a period of time may lead to an accommodation effect indeed, even when using randomized stimulation. However, this problem need to be further investigated to provide accurate answer. Finally, researches on pain will always be biased by the human factor until reliable objective and quantitative pain assessments are developed, thus, robust experimental designs should always try to minimize bias.

\section{Conclusion}

Although using randomized time and intensity stimulation design, we didn't get stable EMG responses along the experiment. We observed that the higher the stimulation intensity, the larger the recognition rates of electromyographic responses for all the three muscles. Furthermore, thermal painful stimulation at the threshold level elicits electromyographic responses in only 20 to $32 \%$ of the total amount of stimuli, while thermal painful stimulation at the tolerance level increase the percentage of electromyographic responses for 54 to $70 \%$ of the total amount of stimuli. Among Corrugator, Zygomaticus and Trapezius muscles, Zygomaticus was the muscle in which responses were more often identified (over $47 \%$ of the time), regardless of the stimulation intensity.

\section{Acknowledgments}

The authors would like to acknowledge the financial support from Brazilian (FAPEMIG, CAPES and CNPq) and German (DFG) agencies.

\section{References}

[1] R. Cowen, M. K. Stasiowska, H. Laycock, and C. Bantel, "Assessing pain objectively: the use of physiological markers," Anaesthesia, p. n/a-n/a, 2015.

[2] S. Walter, S. Gruss, H. Ehleiter, J. Tan, H. C. Traue, S. Crawcour, P. Werner, A. Al-Hamadi, and A. O. Andrade, "The biovid heat pain database data for the advancement and systematic validation of an automated pain recognition system," in 2013 IEEE International Conference on Cybernetics (CYBCO), 2013, pp. 128131.

[3] V. Lichtner, D. Dowding, P. Esterhuizen, S. J. Closs, A. F. Long, A. Corbett, and M. Briggs, "Pain assessment for people with dementia: a systematic review of systematic reviews of pain assessment tools.," BMC Geriatr., vol. 14, p. 138, 2014.

[4] K. M. Prkachin, "Assessing pain by facial expression: Facial expression as nexus," Pain Research and Management, vol. 14, no. 1. pp. 53-58, 2009.

[5] K. Wolf, T. Raedler, K. Henke, F. Kiefer, R. Mass, M. Quante, and K. Wiedemann, "The face of pain - A pilot study to validate the measurement of facial pain expression with an improved electromyogram method," Pain Res. Manag., vol. 10, no. 1, pp. 15-19, 2005.

[6] E. Suzuki, S. Ishigaki, H. Yatani, E. Morishige, and M. Uchida, "Mean power frequency during speech in myalgia patients.," J. Oral Rehabil., vol. 37, no. 9, pp. 692-7, Sep. 2010.

[7] T. Castroflorio, D. Falla, K. Wang, P. Svensson, and D. Farina, "Effect of experimental jaw-muscle pain on 
the spatial distribution of surface EMG activity of the human masseter muscle during tooth clenching," J. Oral Rehabil., vol. 39, no. 11, pp. 81-92, 2012.

[8] W.-G. Yoo, "Comparison of activation and change in the upper trapezius muscle during painful and nonpainful computer work.," J. Phys. Ther. Sci., vol. 27, no. 10, pp. 3283-3284, Oct. 2015.

[9] C. Fernández-de-las-Peñas, D. Falla, L. ArendtNielsen, and D. Farina, "Cervical muscle co-activation in isometric contractions is enhanced in chronic tensiontype headache patients.," Cephalalgia, vol. 28, no. 7, pp. 744-51, Jul. 2008.

[10] P. Reicherts, A. B. M. Gerdes, P. Pauli, and M. J. Wieser, "On the mutual effects of pain and emotion: Facial pain expressions enhance pain perception and vice versa are perceived as more arousing when feeling pain," Pain, vol. 154, no. 6, pp. 793-800, 2013.

[11] R. Luijcks, H. J. Hermens, L. Bodar, C. J. Vossen, J. Van Os, and R. Lousberg, "Experimentally induced stress validated by EMG activity.," PLoS One, vol. 9, no. 4, p. e95215, 2014. 